\title{
DESEMPENHO ACADÊMICO DE DOUTORES EGRESSOS \\ DO PROGRAMA DE PÓS-GRADUAÇÃO EM MEDICINA: CIÊNCIAS MÉDICAS DA FACULDADE DE MEDICINA DA UFRGS
}

\author{
ACADEMIC PERFORMANCE OF DOCTORAL GRADUATES \\ FROM THE GRADUATE PROGRAM IN MEDICINE: \\ MEDICAL SCIENCES AT UFRGS SCHOOL OF MEDICINE
}

\author{
Vera Susana Ribeiro ${ }^{1}$, Lucas França Garcia², \\ Wolnei Caumo ${ }^{1,3}$, Edison Capp ${ }^{1,4}$
}

\section{RESUMO}

Clin Biomed Res. 2020;40(3):154-160

1 Programa de Pós-Graduação em Medicina: Ciências Médicas, Faculdade de Medicina, Universidade Federal do Rio Grande do Sul (UFRGS). Porto Alegre, RS, Brasil.

2 Programa de Pós-Graduação em Promoção da Saúde, UniCesumar. Maringá, PR, Brasil.

3 Departamento de Cirurgia, Faculdade de Medicina, Universidade Federal do Rio Grande do Sul (UFRGS). Porto Alegre, RS, Brasil.

4 Programa de Pós-Graduação em Ciências da Saúde: Ginecologia e Obstetrícia, Faculdade de Medicina, Universidade Federal do Rio Grande do Sul (UFRGS). Porto Alegre, RS, Brasil.

Autor correspondente:

Edison Capp

edcapp@ufrgs.br

Faculdade de Medicina, Universidade

Federal do Rio Grande do Sul (UFRGS)

Rua Ramiro Barcelos, 2400.

90035-003, Porto Alegre, RS, Brasil.
Introdução: embora, no Brasil, muito tenha sido feito para estimular a criação e manutenção de programas de pós-graduação ainda há carência de meios para avaliar e para mensurar a evolução profissional dos egressos. O objetivo é compreender a trajetória dos Doutores formados pelo Programa de Pós-Graduação em Medicina Ciências Médicas (PPGCM), Faculdade de Medicina, Universidade Federal do Rio Grande do Sul (UFRGS) e propor um índice de desempenho acadêmico e de inserção profissional.

Métodos: foi realizado um estudo transversal. Foram coletados dados dos Currículos Lattes dos egressos de doutorado do Programa de Pós-Graduação em Medicina: Ciências Médicas (PPGCM), no período de 1987 a 2014. Foram extraídos apenas os dados pertinentes a sua origem, curso de graduação e de mestrado, produção intelectual, inserção acadêmica e a posição profissional.

Resultados: foram analisados 324 doutores egressos, 221 eram graduados em Medicina. Foram utilizadas as variáveis: graduação em medicina, vínculo laboral em universidade pública, exerce atividade como orientador em nível de doutorado, exerce atividade como orientador em nível de mestrado, bolsista de produtividade em pesquisa do CNPq e possui registro do índice $\mathrm{H}$ no currículo Lattes. O coeficiente de determinação (R2) demonstrou que as variáveis incluídas no modelo explicam $99 \%$ do modelo hierárquico.

Conclusão: os resultados demonstram que os egressos têm contribuído para a construção de conhecimento qualificado disseminado em jornais de circulação internacional, formação de recursos humanos, interagir e criar parcerias em seu ambiente de trabalho, gerenciar e repassar o conhecimento, bem como para fomentar o conhecimento na área das ciências médicas com relevância regional, nacional e internacional.

Palavras-chave: Ensino; pós-graduação; publicações; egressos; doutorado; científico; acadêmico; índice de inserção

\section{ABSTRACT}

Introduction: Although, in Brazil, much has been done to encourage the creation and maintenance of graduate programs, there remains a lack of means to assess and measure the professional outcomes of graduates. The objective is to understand the trajectory of doctoral graduates from the Graduate Program in Medicine: Medical Sciences (PPGCM), School of Medicine, Universidade Federal do Rio Grande do Sul (UFRGS), and propose an index for academic performance and professional placement. 
Methods: A cross-sectional study was conducted. Data available at CV Lattes database of the National Council for Scientific and Technological Development (CNPq) were collected on doctoral graduates from the PPGCM, from 1987 to 2014. Only data pertaining to their origin, undergraduate and master's degrees, intellectual output, academic performance, and professional placement were retrieved.

Results: Data on 324 doctoral graduates were analyzed, 221 had graduated in medicine. Undergraduate degree, employment at a public university, working as a doctoral-level advisor, working as a master's-level advisor, CNPq research productivity grantee, and having a recorded $\mathrm{h}$-index in the Lattes curriculum were collected. The coefficient of determination $\left(R^{2}\right)$ demonstrated that the variables included in the model explain $99 \%$ of the hierarchical model.

Conclusion: The results show that the graduates have contributed to developing qualified knowledge disseminated in international newspapers, training human resources, interacting and creating partnerships in the workplace, managing and passing on knowledge, as well as fostering knowledge in the area of medical sciences with regional, national, and international relevance.

Keywords: Teaching; graduate school; publications; graduates; doctoral degree; scientific; academic; placement index

\section{INTRODUÇÃO}

A pós-graduação stricto sensu tem como objetivo formar recursos humanos qualificados e oportunizar o desenvolvimento de novas tecnologias aplicáveis'. O aprimoramento e titulação docente têm tornado possível o avanço sócio educacional de todo o sistema de graduação e pós-graduação lato e stricto sensu $u^{2,3}$.

Os programas de pós-graduação buscam atender à necessidade de formar pesquisadores, bem como qualificar e aprimorar a área educacional e profissional dos alunos egressos ${ }^{3}$. Esses egressos tornam-se um elo entre a academia e o mercado laboral ao propiciarem que novas tecnologias sejam incorporadas pelas empresas e demais setores da sociedade ${ }^{1}$.

A titulação dos discentes tem propiciado considerável avanço ao sistema de graduação e pós-graduação acadêmica no país ${ }^{3}$. Apesar das dificuldades encontradas na expansão do sistema de ensino no Brasil, a titulação dos discentes tem se mantido robusta pelo apoio, recebido, da Coordenação de Aperfeiçoamento de Pessoal de Nível Superior (Capes) através de diversas linhas de financiamento e apoio a pesquisa (Parecer $\left.n^{\circ} 977 / 65\right)^{4}$. A pósgraduação tem a incumbência de produzir novos conhecimentos, destacando-se o aspecto social e científico, além de, em um país com as dimensões do Brasil, corrigir desigualdades regionais ${ }^{5}$.

O Programa de Pós-Graduação em Medicina: Ciências Médicas (PPGCM) da Faculdade de Medicina da Universidade Federal do Rio Grande do Sul é um programa consolidado e foi criado através do parecer 124/1981 da Câmara de Pós-Graduação (CAMPG/ UFRGS). Esse programa tem caráter abrangente, com ênfase nos aspectos de patogenia das principais moléstias, com áreas básicas de patologia, fisiologia, bioquímica, microbiologia e outras com objetivo de incentivar a produção científica. Ainda não foi examinada a inserção acadêmica de seus egressos. No Brasil, há poucos estudos que abordem o processo de formação e o destino de pesquisadores, considerando especificidades de cada área de conhecimento, formação e campo científico ${ }^{6,7}$. Há necessidade de avaliar o desenvolvimento de carreiras e a adaptação acadêmica dos pós-graduandos.

O objetivo deste estudo foi identificar fatores associados com a inserção no meio cientifico e acadêmico dos doutores egressos do Programa de Pós-Graduação em Medicina Ciências Médicas da Faculdade de Medicina da UFRGS.

\section{MÉTODOS}

\section{Delineamento}

Foi realizado um estudo transversal.

\section{População e amostra}

Foram identificados todos os alunos que concluíram doutorado no Curso de Doutorado no Programa de Pós-Graduação em Medicina: Ciências Médicas (PPGCM), Faculdade de Medicina (Famed), Universidade Federal do Rio Grande do Sul (UFRGS) no período de 1987 (ano de criação do PPGCM) a 2014. Os nomes dos egressos foram utilizados para acessar seus currículos modelo Lattes, o qual é de acesso público. Foi mantida a privacidade e confidencialidade, sendo utilizado 
apenas os dados pertinentes para compreender os objetivos propostos pelo estudo.

Os nomes dos egressos foram obtidos através das atas de defesa de doutorado do PPGCM. A partir disto, foram acessados os Currículos Lattes dos titulados e obtidas as seguintes informações:

- data de atualização do currículo Lattes;

- sexo;

- curso de graduação;

- IES do curso de Graduação;

- IES do curso de Mestrado;

- IES do curso de Doutorado;

- inserção acadêmica, profissional, tecnológica ou industrial;

- número de artigos publicados;

- índice de impacto dos artigos;

- Qualis/Capes dos artigos publicados na área da Medicina I.

Os dados foram coletados da base de dados pública da Plataforma Lattes do Conselho Nacional de Desenvolvimento Científico e Tecnológico (CNPq) e no Sistema de Pós-Graduação (POSGRAD) da Universidade Federal do Rio Grande do Sul.

O projeto foi submetido e aprovado pelo Comitê de Ética e Pesquisa (CEP) da UFRGS (\#42975115.5.0000.5347).

\section{Análise estatística}

A análise descritiva foi realizada por meio da média e desvio padrão, frequência ou proporção. A comparação foi realizada por meio do teste $t$ para amostras independentes foi utilizada para comparação entre médias. Para comparar variáveis categóricas, utilizou-se o teste de qui-quadrado de Pearson ou teste exato de Fischer.

Foi construído um índice ajustado de produção científica e inserção no meio acadêmico profissional utilizando um modelo de regressão. Para construir este índice, foram consideradas as seguintes variáveis: índice de impacto dos periódicos mensurado pelo JCR, idade de conclusão do doutorado, idade do doutorando ao final do último ano e busca (2014), gênero e número de artigos publicados. A partir deste índice utilizado como variável desfecho foram realizadas as análises univariadas para identificar potenciais fatores associados ao desfecho de interesse. Foi realizada a comparação do índice de produção qualificada entre os seguintes fatores referentes aos doutores egressos: curso de graduação, atividade de ensino na graduação, orientações em níveis graduação, especialização, mestrado e doutorado, participação em bancas de avaliação, possuir bolsa de produtividade em pesquisa, registro do índice $\mathrm{H}$ no currículo Lattes, registro na base de dados do Google acadêmico, vínculo laboral. As variáveis que apresentaram significância estatística ao nível de
$5 \%$ foram incluídas no modelo de regressão para identificar os fatores independentes correlacionados com o índice ajustado de produção qualificada. Realizou-se controle para multicolineraidade e o método de entrada das variáveis no modelo foi forwards' stepwise.

No primeiro nível, foram incluídas graduação em medicina ou outros cursos, se possui vínculo docente com universidade pública ou privada e vínculo com qualquer instituição que tenha atividade de ensino. No segundo nível, foram incluídas as variáveis como se estava vinculado a qualquer instituição de ensino, se realiza orientação em níveis de graduação e especialização, mestrado e doutorado. No terceiro nível, foram incluídas as variáveis que podem sofrer influência de todas as demais variáveis incluídas nos modelos precedentes, e mais próximas ao desfecho, tais como existência de registro do índice $\mathrm{H}$ no currículo Lattes, registro no Google acadêmico de acesso público e se possui bolsa de produtividade em pesquisa do CNPq. As variáveis (1) vínculo com qualquer instituição que tenha atividade de ensino, (2) orientação na graduação e especialização e (3) registro no Google acadêmico de acesso público foram excluídas dos modelos por apresentarem colinearidade. Para todas as análises o nível de significância estatística para o erro alfa estabelecido foi um $P<0,05$, bicaudal. As análises foram realizadas por meio do software SPSS versão 21.0 (SPSS, Chicago, IL).

\section{RESULTADOS}

\section{Características sociodemográficas}

No período de 1987 a 2014 foram identificados 324 dos egressos de doutorado do Programa de Pós-Graduação em Medicina: Ciências Médicas da Faculdade de Medicina da UFRGS. Foram incluídos na analise 317 , sete foram excluídos por dados incompletos. A idade dos egressos no momento da defesa foi de $39,49 \pm 8,38$ anos. $47 \%$ dos egressos, foram do sexo feminino $(n=154)$ e $68,8 \%(n=233)$ eram graduados em medicina. As características da amostra estão apresentadas na Tabela 1 e são dados que descrevem a situação dos egressos no momento da realização deste estudo (dados referentes a 2014). $27,5 \%$ eram servidores da Universidade Federal do Rio Grande do Sul, 13,3\% Hospital de Clínicas de Porto Alegre, 8,0\% Universidade Federal de Ciências da Saúde de Porto Alegre, os demais egressos $51,5 \%$ eram de instituições privadas e públicas de ensino/ pesquisa do sistema educacional do país. Sete alunos tinham se graduado no exterior: Argentina (1), Bolívia (2), Estados Unidos (2), Peru (1) e Uruguai (1). Dezesseis realizaram mestrado no exterior (Bélgica, Bolívia, Canadá, Escócia, Estados Unidos, Inglaterra, México e Suíça). 
Tabela 1: Curso de graduação dos egressos.

\begin{tabular}{|c|c|c|}
\hline Característica & Média (SD) & Frequência (\%) \\
\hline \multicolumn{3}{|l|}{ Curso de graduação } \\
\hline Biologia & & $20(6,17 \%)$ \\
\hline Biomedicina & & $5(1,54 \%)$ \\
\hline Educação Física & & $2(0,16 \%)$ \\
\hline Enfermagem & & $10(3,09 \%)$ \\
\hline Farmácia/Bioquímica & & $36(11,11 \%)$ \\
\hline Fisioterapia & & $8(2,46 \%)$ \\
\hline Fonoaudiologia & & $4(1,23 \%)$ \\
\hline Medicina & & $221(68,2 \%)$ \\
\hline Nutrição & & $8(2,46 \%)$ \\
\hline Odontologia & & $3(0,92 \%)$ \\
\hline Psicologia & & $3(0,92 \%)$ \\
\hline Física & & $1(30 \%)$ \\
\hline Veterinária & & $3(0,92 \%)$ \\
\hline Medicina & & $218(67,28 \%)$ \\
\hline Vínculo laboral com instituição de ensino pública & & $239(73,76 \%)$ \\
\hline Vínculo laboral com instituição de ensino privada & & $79(24,38 \%)$ \\
\hline Exerce atividade de em instituições de ensino e ou pesquisa & & $297(91,66 \%)$ \\
\hline Exerce atividade de orientação na graduação ou especialização & & $197(60,8 \%)$ \\
\hline Exerce atividade como orientador de mestrado & & $137(42,28 \%)$ \\
\hline Exerce atividade como orientador de doutorado & & $85(26,23 \%)$ \\
\hline Participação em bancas examinadoras & & $258(60,8 \%)$ \\
\hline Possui registro do índice $\mathrm{H}$ no currículo Lattes & & $87(26,85 \%)$ \\
\hline Possui registro na Plataforma do Google Acadêmico & & $30(9,25 \%)$ \\
\hline Bolsista de produtividade em Pesquisa do CNPq & & $35(10,8 \%)$ \\
\hline Número de artigos publicados & $42,06(52,36)$ & \\
\hline Número de artigos publicados em Jornais com JCR & $13,27(18,54)$ & \\
\hline
\end{tabular}

Os cursos de graduação mais frequentes dos doutores egressos do PPGCM foram: Medicina $(68,2 \%)$, Farmácia $(10,5 \%)$, Biologia $(6,2 \%)$, Enfermagem (3,1\%) e Fisioterapia (2,5\%) (Tabela 1$)$. Dentre os egressos, $49,4 \%$ foram graduados na UFRGS, $8,0 \%$ na Pontifícia Universidade Católica do Rio Grande do Sul (PUCRS), 8,0\% da Universidade Federal de Ciências da Saúde de Porto Alegre (UFCSPA) e $6,5 \%$ da Universidade Federal de Santa Maria (UFSM).

\section{Produção qualificada classificada pelo critério Qualis/Capes}

As classificações Qualis/Capes dos periódicos em que os artigos foram publicados são apresentadas na Figura 1. O Fator de Impacto médio das revistas foi 3,16 $\pm 4,88$, com mediana 2,14 quartil (Q25-75; 1,03 a 3,53). Na Figura 1 está apresentada apresenta as frequências dos artigos publicados pelos egressos nos estratos Qualis/Capes. 18,4\% possuem publicações em periódicos de Qualis A1, $17,3 \%$ em A2, $28,31 \%$ em B2 e $27,49 \%$ em B1, perfazendo um total de $91,55 \%$ da amostra. Os demais $8,46 \%$ serão encontrados em periódicos de impacto e indexação B3 e B4.
Foi possível verificar que entre os alunos de doutorado da $30 \%$ dos egressos possuem até 5 artigos publicados, $27,3 \%$ possui de 6 a 10 artigos publicados e $16,3 \%$ possuem de 11 a 20 artigos publicados. $9,8 \%$ dos doutores possuem de 31 a 50 artigos publicados.

$\mathrm{Na}$ Tabela 2 estão apresentadas as médias (DP) do índice ajustado de produção qualificada. $\mathrm{Na}$ análise univariada, observou-se diferença na comparação do índice de produção qualificada nos seguintes fatores: curso de graduação, vínculo laboral, atividade de orientação, participação em bancas examinadoras, registro do índice $\mathrm{H}$ ou na base de dados do Google Acadêmico, bolsista de produtividade em pesquisa.

Para obtenção do efeito independente no índice de produção qualificada o escore do mesmo foi ajustado usando um modelo de regressão linear múltipla (Tabela 3). Os egressos com graduação em medicina, registro de bolsa de produtividade em Pesquisa do CNPq, exerce atividades de orientação a alunos de graduação, especialização, mestrado ou doutorado e registro do índice $\mathrm{H}$ no currículo Lattes. O conjunto de variáveis incluídas no modelo explica 99\% da variância no índice de produção qualificada no meio acadêmico e profissional. 


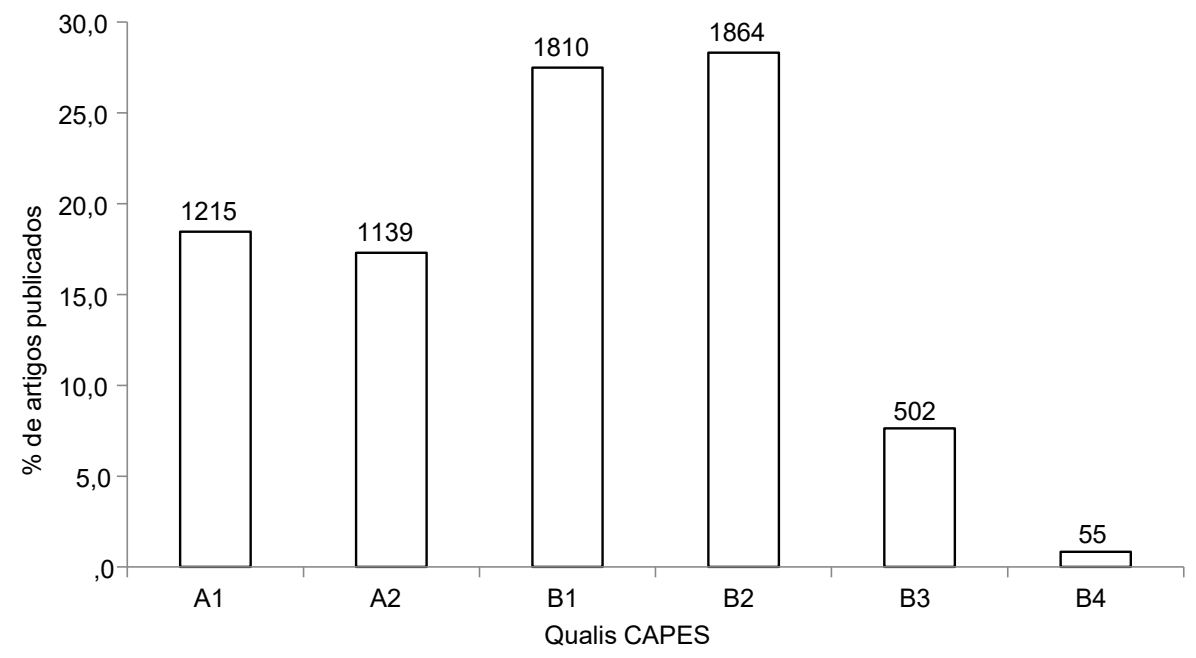

Figura 1: Qualis/Capes dos artigos publicados no período de 1987 a 2014.

Tabela 2: Análise univariada da comparação de médias entre os potencias fatores associados com o índice de produção qualificada, Dados apresentados como média \pm DP $(n=317)$.

\begin{tabular}{|c|c|c|c|}
\hline \multicolumn{4}{|l|}{ Desfecho: índice de produção qualificada } \\
\hline \multirow[t]{2}{*}{ Característica } & \multicolumn{2}{|c|}{ Média (SD) } & \multirow[t]{2}{*}{$\mathbf{P}$} \\
\hline & Sim & Não & \\
\hline Curso de graduação em medicina & $0,48(0,50)$ & $0,24(027)$ & 0,001 \\
\hline Exerce atividade laboral em instituição com atividades de ensino & $0,41(0,34)$ & $0,31(0,26)$ & 0,1 \\
\hline Vínculo laboral com instituição de ensino pública & $0,47(0,50)$ & $0,23(0,17)$ & 0,001 \\
\hline Exerce atividade como orientador na graduação ou especialização & $0,51(0,37)$ & $0,24(0,18)$ & 0,001 \\
\hline Exerce atividade como orientador de mestrado ou doutorado & $0,69(0,33)$ & $0,19(0,09)$ & 0,001 \\
\hline Participação em bancas examinadoras & $0,46(0,48)$ & $0,16(0,12)$ & 0,001 \\
\hline Possui registro do índice $\mathrm{H}$ no currículo Lattes & $0,71(0,59)$ & $0,29(0,32)$ & 0,001 \\
\hline Possui registro na Plataforma do Google Acadêmico & $0,70(0,57)$ & $0,37(0,43)$ & 0,005 \\
\hline Bolsista de produtividade em Pesquisa do CNPq & $1,11(0,65)$ & $0,32(0,33)$ & 0,001 \\
\hline
\end{tabular}

Tabela 3: Modelo hierárquico de regressão linear múltipla para ajustar o escore do índice de produção qualificada no meio acadêmico e profissional aos potencias confundidores $(n=317)$.

\begin{tabular}{|c|c|c|c|c|c|c|}
\hline \multicolumn{7}{|l|}{ Desfecho: Índice de produção qualificada } \\
\hline & B & Erro padrão & $\mathbf{B}^{\Sigma}$ & $\mathbf{t}$ & $\mathbf{P}$ & IC $95 \%$ \\
\hline Graduação em medicina $†$ & 0,199 & 0,038 & 0,272 & 5,180 & 0,00 & $(0,12$ a 0,27$)$ \\
\hline Vínculo laboral em universidade pública $†$ & 0,196 & 0,041 & 0,250 & 4,758 & 0,00 & $(0,11$ a 0,28$)$ \\
\hline Exerce atividade como orientador em nível de doutorado§ & 0,525 & 0,025 & 0,685 & 21,402 & 0,00 & $(0,48$ a 0,57$)$ \\
\hline Exerce atividade como orientador em nível de mestrado§ & 0,170 & 0,022 & 0,248 & 7,814 & 0,00 & $(0,13$ a 0,21$)$ \\
\hline Bolsista de produtividade em Pesquisa do CNPq\|l & 0,435 & 0,002 & 0,401 & 225,764 & 0,00 & $(0,43$ a 0,44$)$ \\
\hline Possui registro do índice $\mathrm{H}$ no currículo Lattes|| & 0,121 & 0,001 & 0,160 & 92,972 & 0,00 & $(0,11$ a 0,12$)$ \\
\hline
\end{tabular}

$\Sigma$ Coeficiente padronizado, R2 ajustado= 0,99; † Modelo 1: graduação, tipo de vínculo e universidade pública ou privada; $\S$ Modelo 2: modelo 1 ensino, orientação em níveis de graduação, especialização, mestrado e doutorado; || Modelo 3: modelo 2 mais índices $\mathrm{H}$, registro no Google acadêmico de acesso público, bolsista de produtividade do CNPq.

\section{DISCUSSÃO}

O presente estudo objetivou identificar a trajetória e a inserção no meio científico e acadêmico dos doutores egressos do Programa de Pós-Graduação em Medicina Ciências Médicas da Faculdade de Medicina da UFRGS, no período que compreende 1987 a 2014, e a partir disto propor um índice de desempenho acadêmico. Foram identificados como fatores correlacionados ao índice de produção qualificada do meio acadêmico e profissional: vínculo laboral em universidade pública, possuir bolsa de produtividade em pesquisa do CNPq, exercer atividade de orientação de trabalhos em níveis de mestrado ou doutorado, ter graduação na área da saúde, possuir registro do índice $\mathrm{H}$ no Currículo Lattes.

De acordo com os estratos Qualis/Capes, $91,55 \%$ possuem artigos publicados entre os estratos $\mathrm{A} 1 \mathrm{a}$ 
B2 da área da Medicina I. A publicação em jornais de maior impacto possibilita aumento da visibilidade com consequente aumento no índice de citações ${ }^{8}$. Os alunos egressos apresentam publicações predominantemente em revistas de alto impacto o que demonstra a qualificação dos cientistas formados. Considerando que a avaliação dos programas de pós-graduação pela Capes considera o acompanhamento dos egressos, há necessidade da criação de um indicador qualitativo de performance. Este deve medir e avaliar a qualidade de formação dos doutores egressos e também servir como parâmetro que auxilie a promover uma melhoria contínua no processo formativo. Trata-se de um índice com potencial para indicar qualidade, com definição das dimensões da métrica para garantir a sua operacionalização e relevância.

Os resultados do presente estudo evidenciam que os doutores egressos do PPGCM, aqui definido como produto, têm alcançado boa inserção no meio acadêmico e profissional, usando para isso como referência a produção qualificada. Dentre os egressos, $239(73,76 \%)$ atuam em instituições de ensino públicas e 297 (91,66\%) atuam em instituições com vínculo em atividade de ensino. Formar pessoas qualificadas refletirá no aprimoramento das áreas de conhecimento com maior qualidade e relevância para o mercado de nosso país ${ }^{1}$.

Este índice foi construído como um indicador qualitativo dos egressos de doutorado do PPGCM ao longo do tempo. Embora seja difícil estabelecer a melhor medida do perfil de egressos, o conjunto de fatores selecionados na construção de nosso modelo hierárquico não privilegia apenas o processo da métrica apenas pela produção de artigos, nem se sustenta no impacto dos jornais ondes os mesmos foram publicados. Trata-se de um índice construído a partir de um conjunto de variáveis acessíveis e que apresentam certa fidedignidade, com vistas à construção de um índice com amplo espectro que considere também sua atuação profissional. Observa-se que o conjunto de variáveis explica $99 \%$ da variância de nosso modelo, o que sugere que o conjunto de parâmetros utilizados constitui uma métrica avaliativa consistente. Embora a construção deste índice métrico de desempenho envolva um conjunto de fatores não completamente separáveis, este modelo foi construído a partir de um racional que considera aspectos demográficos, a inserção no mercado de trabalho, a atuação no processo de formação de recursos humanos, bem como métricas que se relacionam a qualidade do produto avaliado pelo índice de citações. $O$ fator de impacto demonstra a influência ou a qualidade da publicação (artigo ou revista) e consequentemente de seus autores, cursos e instituições ${ }^{8}$. Esta estratégia oportuniza uma avaliação ampla, com a inclusão das múltiplas facetas do processo formativo.

Entende-se que se trata de um processo complexo, onde mesmo usando artifícios da estatística não é possível considerar que os fatores inclusos em cada um dos modelos dentro de uma visão hierárquica sejam absolutamente independentes uma vez que se mostram entrelaçados e em uma relação do tipo causa e consequência. Reconhece-se a necessidade de criar nos egressos uma cultura de valorização das informações sobre sua atuação profissional pós titulação, tendo em vista a importância que a Comissão de Avaliação da Capes atribui a este item ${ }^{9}$. Observouse que os profissionais titulados são valorizados no mercado, pois estão inseridos em instituições de ensino superior pública e privado de excelência no mercado.

Os fatores utilizados na construção deste índice permitem classificar as características, resultados e consequências das ações dos egressos por meio de um valor numérico, aqui denominado de índice. Os atributos utilizados na construção deste índice são de fácil coleta e o mesmo é passível de ser compreendido de maneira simples uma vez que traduz em resultados efetivos a trajetória e contribuição científica destes egressos. O índice aqui proposto permite formar uma série histórica, contextualizada, que evidencia a evolução do desempenho dos egressos do PPGCM, Famed, UFRGS. Trata-se de um indicador de efetividade e impacto, por se vincular as transformações produzidas pela atuação dos doutores egressos nos diferentes contextos do ensino, pesquisa e assistência. Este índice pode auxiliar nos processos decisórios, e estabelecer uma sistemática de monitoramento e avaliação da formação de recursos humanos na pós-graduação.

Entre as limitações deste estudo, destaca-se que o Currículo Lattes é um documento de preenchimento voluntário, muitas vezes desatualizado e incompleto. Em estudos futuros, a busca da produção científicoacadêmica, assim como o seu impacto, pode ser ampliada incluindo outras bases de dados (PubMed; ISI Web of Science, Scopus, por exemplo).

Os resultados demonstram que um grande número de egressos se vinculou a alguma instituição de ensino, desenvolvendo uma carreira acadêmica, promovendo formação de recursos humanos e de produção científica. Isto pode ser evidenciado pelas publicações em jornais científicos de circulação internacional, formação de recursos humanos, interação e criação de parcerias em seu ambiente de trabalho, geração de conhecimento e fomento do conhecimento na área das ciências médicas com relevância regional, nacional e internacional.

\section{Agradecimentos}

Edison Capp e Wolnei Caumo são bolsistas de produtividade em pesquisa do Conselho Nacional de Desenvolvimento Científico e Tecnológico (CNPq). Este artigo é resultado do mestrado de Vera Susana Vargas Ribeiro. A dissertação pode ser acessada em: https://bit.ly/2ALqVc9. 


\section{REFERÊNCIAS}

1. Velho L. O papel da formação de pesquisadores no sistema de inovação. Cienc Cult. 2007;59(4):23-32.

2. Guimarães JA. A pesquisa médica e biomédica no Brasil: comparações com o desempenho científico brasileiro e mundial. Cien Saude Colet. 2004;9(2):303-27.

3. Guimarães R. Pesquisa no Brasil: a reforma tardia. Sao Paulo Perspec. 2002;16:(4)41-7.

4. Conselho de Educação Superior (BR) Parecer $n^{\circ}$ 977, de 3 de dezembro de 1965. Diário Oficial da União
[Internet]. 1965 Dez 3 [cited 2020

Nov 11]. Available from: http://cad. capes.gov.br/ato-administrativodetalhar?idAtoAdmElastic $=314$

5. Cirani CBS, Campanario MA, Silva HHM. A evolução do ensino da pós-graduação senso estrito no Brasil: análise exploratória e proposições para pesquisa. Avaliação. 2015;20(1):163-7.

6. Louzada RCR, Silva Filho JF. Pósgraduação e trabalho: um estudo sobre projetos e expectativas de doutorandos brasileiros. Hist Cienc Saude Manguinhos. 2005;12(2):265-82.
7. Schanaider A. Sistema de mapeamento dos egressos. Rev Col Bras Cir. 2015;42(6):413-7.

8. Bicas HEA, Rother ET, Braga MER. Fatores de impacto, outros índices bibliométricos e desempenhos acadêmicos. Arq Bras Oftalmol. 2002;65(2):151-2.

9. Ortigoza SAG, Poltronieri LC, Machado LMCP. A atuação profissional dos egressos como importante dimensão no processo de avaliação de programas de pós-graduação. Soc Nat. 2012;24(2):243-54.

Recebido: 17 maio, 2019 Aceito: 3 out, 2020 